\title{
GRAPHS WHOSE FULL AUTOMORPHISM GROUP IS A SYMMETRIC GROUP
}

\author{
MARTIN W. LIEBECK \\ (Received 16 May 1986; revised 5 September 1986) \\ Communicated by L. Caccetta
}

\begin{abstract}
We address the problem of describing all graphs $\Gamma$ such that Aut $\Gamma$ is a symmetric group, subject to certain restrictions on the sizes of the orbits of Aut $\Gamma$ on vertices. As a corollary of our general results, we obtain a classification of all graphs $\Gamma$ on $v$ vertices with Aut $\Gamma \cong S_{n}$, where

$$
v<\min \left\{5 n, \frac{1}{2} n(n-1)\right\} \text {. }
$$
\end{abstract}

1980 Mathematics subject classification (Amer. Math. Soc.): 05 C 25.

\section{Introduction}

It has been known since Frucht's paper [1] of 1938 that, given any finite group $G$, there is a graph $\Gamma$ such that the automorphism group of $\Gamma$ is isomorphic to $G$. For certain groups $G$, such as $S_{n}$, this result is obvious, and it is more interesting to investigate the more general problem of describing all graphs $\Gamma$ such that Aut $\Gamma \cong G$. In this paper we address this problem for the symmetric groups $S_{n}$. This was considered for graphs with less than $2 n$ vertices in [2] and [3]. Here we investigate the graphs $\Gamma$ such that Aut $\Gamma \cong S_{n}$, under the following far less restrictive hypothesis:

all orbits of Aut $\Gamma$ on the set $V \Gamma$ of vertices of $\Gamma$ have size less than $\frac{1}{2} n(n-1)$.

(C) 1988 Australian Mathematical Society $0263-6115 / 88$ \$A2.00 +0.00 
It is an elementary consequence of $(*)$ (see Proposition 1.2 below) that for $n>6$, all the orbits of Aut $\Gamma$ on $V \Gamma$ have size 1 or $n$. Let $t$ be the number of orbits of size $n$. In Theorem 1.4 we show that $\Gamma$ must satisfy various strong necessary conditions; and we conjecture (1.5) that these conditions on an arbitrary graph $\Delta$ are also sufficient to imply that Aut $\Delta \cong S_{n}$. We prove Conjecture 1.5 for $1 \leqslant t \leqslant 4$ (see Theorem 2.7). In particular this gives a classification of all graphs $\Gamma$ with Aut $\Gamma \cong S_{n}(n>6)$ and $|V \Gamma|<\min \left\{5 n, \frac{1}{2} n(n-1)\right\}$. This substantially improves the results of [2] and [3], and also solves various problems raised in [3, Section 4]. We include general descriptions of these graphs in an Appendix.

Finally, we remark that the methods of this paper will extend to the analysis of graphs with automorphism group $S_{n}$ under weaker hypotheses than $(*)$ (see Remark 3 after Theorem 2.7).

Notation. If $G$ is a permutation group on a set $\Omega$ and $\Delta \subseteq \Omega$ then $G_{\{\Delta\}}$ denotes the setwise stabilizer of $\Delta$ in $G$; and if $\Psi$ is a fixed set of $G$ then $G^{\Psi}$ denotes the action of $G$ on $\Psi$. Also $\operatorname{Alt}(\Omega)$ and $\operatorname{Sym}(\Omega)$ denote, respectively, the alternating and symmetric groups on $\Omega$.

\section{A general result and a conjecture}

We begin with an elementary proposition.

Proposition 1.1. Let $n>6$ and let $H$ be a proper subgroup of $S_{n}$ with $\left|S_{n}: H\right|<\frac{1}{2} n(n-1)$. Then $H$ is $A_{n}, S_{n-1}$ or $A_{n-1}$.

Proof. If $H$ is transitive and imprimitive on the $n$ points with blocks of size $a$ and $a b=n(a \neq 1, b \neq 1)$, then $|H| \leqslant(a !)^{b} b$ !, so

$$
\frac{1}{2} n(n-1)>\left|S_{n}: H\right| \geqslant n ! /\left((a !)^{b} b !\right)
$$

which forces $n \leqslant 6$, a contradiction. If $H$ is primitive on the $n$ points and $H \neq A_{n}$ then a result of Bochert (Theorem 14.2 of [4]) gives $\frac{1}{2} n(n-1)>\left|S_{n}: H\right|$ $\geqslant\left[\frac{1}{2}(n+1)\right]$ !, forcing $n \leqslant 6$ or $n=8$. The latter is impossible (an easy check) so this case cannot occur. Finally if $H$ is intransitive with an orbit of size $r$ then $\frac{1}{2} n(n-1)>\left|S_{n}: H\right| \geqslant\left(\begin{array}{c}n \\ r\end{array}\right)$, so that $r$ is 1 or $n-1$ and $H$ is $S_{n-1}$ or $A_{n-1}$.

From Proposition 1.1 we see that if $\Gamma$ is a graph with Aut $\Gamma \cong S_{n}(n>6)$ and all orbits of Aut $\Gamma$ on $V \Gamma$ have size less than $\frac{1}{2} n(n-1)$ then these orbit sizes all lie in $\{1,2, n, 2 n\}$. We shall easily show below (Proposition 1.2) that the orbit 
sizes 2 and $2 n$ cannot occur, so for the remainder of this section we concentrate on the set $\mathscr{E}_{n}$ of graphs defined as follows:

Definition. Let $\Gamma$ be a graph and let $n \geqslant 2$. Then $\Gamma \in \mathscr{E}_{n}$ if and only if Aut $\Gamma$ has a subgroup $G$ isomorphic to $S_{n}$ such that all orbits of $G$ on $V \Gamma$ have size 1 or $n$.

Let $\Gamma \in \mathscr{E}_{n}$. Then we may take $\Gamma$ to be a graph on $t n+r$ vertices $\left\{\alpha_{11}, \ldots, \alpha_{1 n}, \ldots, \alpha_{t 1}, \ldots, \alpha_{t n}, \phi_{1}, \ldots, \phi_{r}\right\}$ such that Aut $\Gamma$ has a subgroup $G$ isomorphic to $S_{n}$ with $r$ fixed points $\phi_{1}, \ldots, \phi_{r}$ and $t$ orbits $\Delta_{1}, \ldots, \Delta_{t}$ of size $n$, where $\Delta_{i}=\left\{\alpha_{i 1}, \ldots, \alpha_{i n}\right\}(i=1, \ldots, t)$. It is clear that each subgraph $\Delta_{i}$ is either the complete graph $K_{n}$ or the null graph $V_{n}$ and that for any $i, j, \phi_{j}$ is joined to all or no vertices in $\Delta_{i}$. For any $i, j, k$ define

$$
\Gamma_{j}\left(\alpha_{i k}\right)=\left\{\alpha_{j l} \in \Delta_{j} \mid \alpha_{j l} \text { is joined to } \alpha_{i k} \text { in } \Gamma\right\} .
$$

Then $\Gamma_{j}\left(\alpha_{i k}\right)$ is a union of orbits of the stabiliser $G_{a_{i k}}$ on $\Delta_{j}$. Now if $n \neq 6$ then $S_{n}$ has just one conjugacy class of subgroups of index $n$, so we may assume in this case that $G_{\alpha_{i k}}=G_{\alpha_{j k}}$ for all $i, j, k$; and $S_{6}$ has two conjugacy classes of subgroups of index 6 , one class containing the stabilizer of one of the 6 points and the other containing a subgroup $S_{5}$ transitive on the 6 points. Hence for any $n \geqslant 2$ and any $i, j, k$ we may assume that $G_{\alpha_{i k}}$ is either transitive on $\Delta_{j}$ or has orbits $\left\{\alpha_{j k}\right\}$ and $\Delta_{j} \backslash\left\{\alpha_{j k}\right\}$ on $\Delta_{j}$. Consequently $\Gamma_{j}\left(\alpha_{i k}\right)$ is one of the sets $\varnothing, \Delta_{j}$, $\left\{\alpha_{j k}\right\}$ and $\Delta_{j} \backslash\left\{\alpha_{j k}\right\}$; and for any $k, l$, if $\Gamma_{j}\left(\alpha_{i k}\right)$ is $\varnothing\left(\Delta_{j},\left\{\alpha_{j k}\right\}, \Delta_{j} \backslash\left\{\alpha_{j k}\right\}\right)$ then $\Gamma_{j}\left(\alpha_{i l}\right)$ is $\varnothing\left(\Delta_{j},\left\{\alpha_{j l}\right\}, \Delta_{j} \backslash\left\{\alpha_{j l}\right\}\right.$ respectively).

Note that the above analysis goes through if we replace $S_{n}$ by the alternating group $A_{n}$. Using this analysis we now prove the result promised above.

Proposition 1.2. Let $\Gamma$ be a graph with Aut $\Gamma \cong S_{n}(n>6)$ and suppose that all orbits of Aut $\Gamma$ on $V \Gamma$ have size less than $\frac{1}{2} n(n-1)$. Then all these orbits have size 1 or $n$.

Proof. Write $G=$ Aut $\Gamma$. By Proposition 1.1 all $G$-orbits on $V \Gamma$ have size 1, 2 , $n$ or $2 n$. Let $H<G$ with $H \cong A_{n}$. Then all $H$-orbits on $V \Gamma$ have size 1 or $n$; let those of size $n$ be $\Delta_{1}, \ldots, \Delta_{t}$ and let fix $H=\left\{\phi_{1}, \ldots, \phi_{r}\right\}$. By the above analysis, each $\Delta_{i}$ is $K_{n}$ or $V_{n}$, each $\phi_{j}$ is joined to all or no vertices of $\Delta_{i}$ and, writing $\Delta_{i}=\left\{\alpha_{i 1}, \ldots, \alpha_{i n}\right\}$, we may choose notation so that $\Gamma_{j}\left(\alpha_{i k}\right)$ is one of $\varnothing, \Delta_{j}$, $\left\{\alpha_{j k}\right\}$ and $\Delta_{j} \backslash\left\{\alpha_{j k}\right\}$ and if $\Gamma_{j}\left(\alpha_{i k}\right)$ is $\varnothing\left(\Delta_{j},\left\{\alpha_{j k}\right\}, \Delta_{j} \backslash\left\{\alpha_{j k}\right\}\right)$ then $\Gamma_{j}\left(\alpha_{i l}\right)$ is $\varnothing\left(\Delta_{j},\left\{\alpha_{j l}\right\}, \Delta_{j} \backslash\left\{\alpha_{j l}\right\}\right.$ respectively) (any $\left.i, j, k, l\right)$. It is clear from this that Aut $\Gamma$ contains a subgroup $K$ such that $K \cong S_{n}, K$ has orbits $\Delta_{1}, \ldots, \Delta_{t}$ and fixes each $\phi_{j}$. Hence $K=G$ and the orbits of $G$ all have size 1 or $n$. 
We now resume our analysis of a graph $\Gamma \in \mathscr{E}_{n}$ on vertex set $\Delta_{1} \cup \cdots \cup \Delta_{t} \cup$ $\left\{\phi_{1}, \ldots, \phi_{r}\right\}$ as described above. From $\Gamma$ we define a coloured graph $\Gamma^{*}$ with vertex set $\left\{\delta_{1}, \ldots, \delta_{t}, \phi_{1}, \ldots, \phi_{r}\right\}$ having 3 vertex-colours (white, black and red) and 5 edge-colours $(0,1, n-1, n$ and black) as follows:

(i) $\delta_{i}$ is coloured white if $\Delta_{i}$ is $V_{n}$, black if $\Delta_{i}$ is $K_{n}(i=1, \ldots, t)$;

(ii) $\phi_{j}$ is coloured red $(j=1, \ldots, r)$;

(iii) $\phi_{j}$ is joined to $\delta_{i}$ by a black edge if $\phi_{j}$ is joined in $\Gamma$ to all vertices of $\Delta_{i}$, and by no edge at all if not;

(iv) $\phi_{i}$ is joined to $\phi_{j}$ by a black edge if $\phi_{i}$ is joined to $\phi_{j}$ in $\Gamma$, and by no edge if not;

(v) the vertices $\delta_{i}, \delta_{j}$ are joined by an edge coloured $0,1, n-1$ or $n$ as follows (if $n=2$ then the labels 1 and $n-1$ should represent different colours):

$$
\begin{aligned}
& \delta_{i} \times \frac{0}{0} \times \delta_{j} \quad \text { if } \Gamma_{j}\left(\alpha_{i k}\right)=\varnothing, \\
& \delta_{i} \times 1-1 \times \delta_{j} \quad \text { if } \Gamma_{j}\left(\alpha_{i k}\right)=\left\{\alpha_{j k}\right\} \text {, } \\
& \delta_{i} \times \stackrel{\mathrm{n}-1}{ } \times \delta_{j} \quad \text { if } \Gamma_{j}\left(\boldsymbol{\alpha}_{i k}\right)=\Delta_{j} \backslash\left\{\alpha_{j k}\right\}, \\
& \delta_{i} \times \frac{n}{n} \times \delta_{j} \quad \text { if } \Gamma_{j}\left(\alpha_{i k}\right)=\Delta_{j},
\end{aligned}
$$

(here $\times$ represents a black or a white vertex). The automorphism group Aut $\Gamma^{*}$ is the group of permutations of $V \Gamma^{*}$ preserving all vertex- and edge-colours. Clearly $\Gamma$ can be reconstructed from $\Gamma^{*}$; so $\Gamma \leftrightarrow \Gamma^{*}$ is a 1-1 correspondence.

Now we define two further graphs from $\Gamma^{*}$ : firstly, $\Gamma_{0}^{*}$ is the subgraph of $\Gamma^{*}$ on $\delta_{1}, \ldots, \delta_{t}$ with all edges coloured 0 or $n$ deleted and all edges coloured 1 or $n-1$ replaced by a black edge; secondly, $\Gamma_{1}^{*}$ is obtained from $\Gamma^{*}$ by the following replacements:

(1) replace all vertices $\delta_{i}, \phi_{j}$ by black vertices $\delta_{i}, \phi_{j}$;

(2) replace any edge coloured 1 or $n$ by a black edge;

(3) delete any edge coloured 0 or $n-1$.

Thus $\Gamma_{1}^{*}$ can be regarded as an uncoloured graph.

We aim to obtain necessary and sufficient conditions for Aut $\Gamma \cong S_{n}$ purely in terms of the smaller graphs $\Gamma^{*}, \Gamma_{0}^{*}$ and $\Gamma_{1}^{*}$.

EXAMPLE. If $\Gamma^{*}$ is

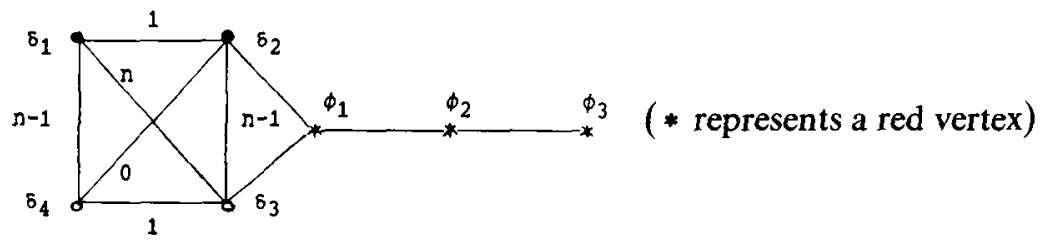


50

Martin W. Liebeck

[5]

then $\Gamma_{0}^{*}, \Gamma_{1}^{*}$ are respectively

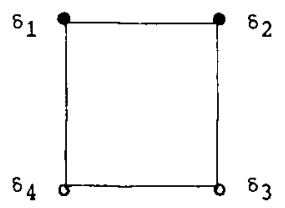

and

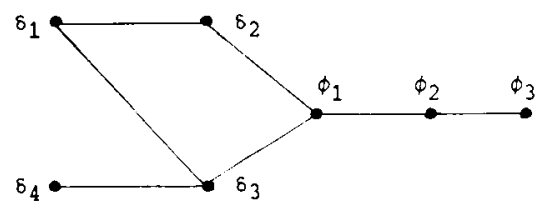

Now consider a coloured graph $\Gamma^{*}$ with $t=r=2$, that is, with $V \Gamma^{*}=$ $\left\{\delta_{1}, \delta_{2}, \phi_{1}, \phi_{2}\right\}$. Let $\Gamma$ be the corresponding graph in $\mathscr{E}_{n}$ with $V \Gamma=\Delta_{1} \cup \Delta_{2} \cup$ $\left\{\phi_{1}, \phi_{2}\right\}$. It is easy to see that Ant $\Gamma$ contains a subgroup isomorphic to $S_{n+1}$ having orbits $\Delta_{1} \cup\left\{\phi_{i_{1}}\right\}$ and $\Delta_{2} \cup\left\{\phi_{i_{2}}\right\}$ (where $\left\{i_{1}, i_{2}\right\}=\{1,2\}$ ) on $V \Gamma$ if and only if $\Gamma^{*}$ is isomorphic to one of the following 12 graphs:
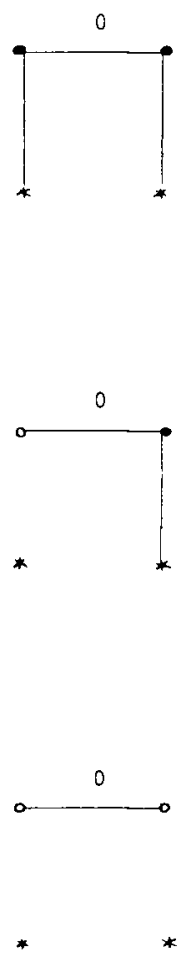
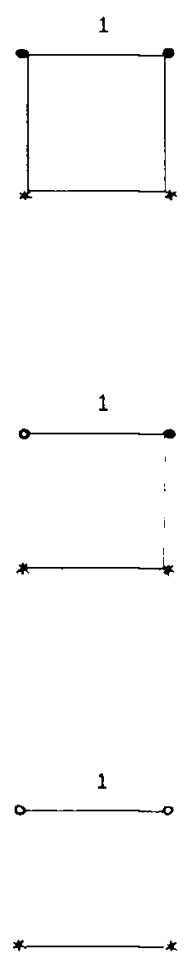
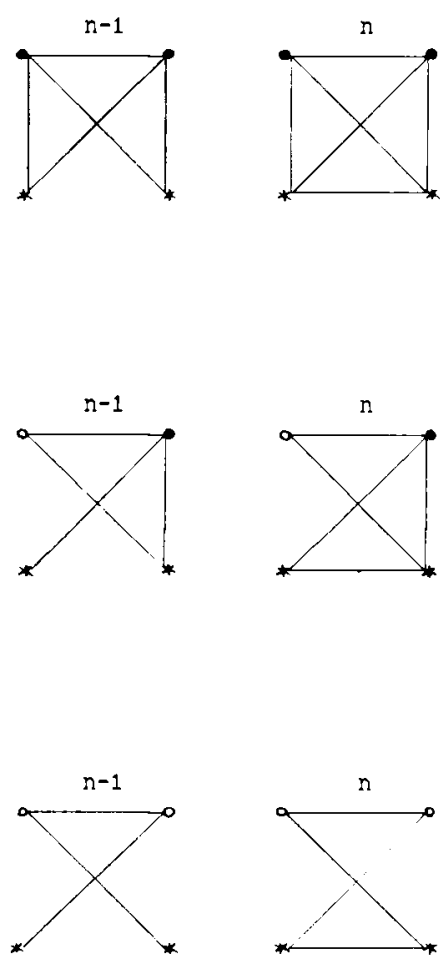

https://doi.org/10.1017/S1446788700031360 Published online by Cambridge University Press 
(where $\circ, \cdot, *$ represent white, black and red vertices respectively). Denote this set of 12 graphs by $\mathscr{C}_{n}$. Note that the graphs in $\mathscr{C}_{n}$ correspond to 6 graphs in $\mathscr{E}_{n}$ and their complements.

LEMma 1.3. Let $\Gamma \in \mathscr{E}_{n}$ have vertex set $\Delta_{1} \cup \cdots \cup \Delta_{t} \cup\left\{\phi_{1}, \ldots, \phi_{r}\right\}$ as above and let $\Gamma^{*}$ and $\Gamma_{1}^{*}$ be the graphs corresponding to $\Gamma$ as above. Suppose that Aut $\Gamma_{1}^{*}$ contains an automorphism $x=\left(\delta_{1} \phi_{i_{1}}\right) \cdots\left(\delta_{t} \phi_{i_{t}}\right)\left(i_{1}, \ldots, i_{t}\right.$ all distinct $)$ such that

(i) $\phi_{i_{j}}$ is joined to $\delta_{j}$ in $\Gamma^{*}$ if and only if $\delta_{j}$ is black $(j=1, \ldots, t)$, and

(ii) for any distinct, $k$, l the subgraph $\left\{\delta_{k}, \delta_{l}, \phi_{i_{k}}, \phi_{i_{l}}\right\}$ of $\Gamma^{*}$ lies in the set $\mathscr{C}_{n}$ of 12 graphs defined above.

Then $\Gamma \in \mathscr{E}_{n+1}$.

Proof. For $j=1, \ldots, t$ put $\Delta_{j}^{\prime}=\Delta_{j} \cup\left\{\phi_{i_{j}}\right\}$. By (i) each subgraph $\Delta_{j}^{\prime}$ is either $K_{n+1}$ or $V_{n+1}$. Write $\phi_{i_{j}}=\alpha_{j, n+1}(j=1, \ldots, t)$ and for any $i, j, k$ define

$$
\Gamma_{j}^{\prime}\left(\alpha_{i k}\right)=\left\{\alpha_{j l} \in \Delta_{j}^{\prime} \mid \alpha_{j l} \text { is joined to } \alpha_{i k} \text { in } \Gamma\right\} .
$$

Then by (i) and (ii), $\Gamma_{j}^{\prime}\left(\alpha_{i k}\right)$ is one of the sets $\varnothing, \Delta_{j}^{\prime},\left\{\alpha_{j k}\right\}$ and $\Delta_{j}^{\prime} \backslash\left\{\alpha_{j k}\right\}$ and for any $k, l$, if $\Gamma_{j}^{\prime}\left(\alpha_{i k}\right)$ is $\varnothing\left(\Delta_{j}^{\prime},\left\{\alpha_{j k}\right\}, \Delta_{j}^{\prime} \backslash\left\{\alpha_{j k}\right\}\right)$ then $\Gamma_{j}^{\prime}\left(\alpha_{i l}\right)$ is $\varnothing\left(\Delta_{j}^{\prime},\left\{\alpha_{j l}\right\}\right.$, $\Delta_{j}^{\prime} \backslash\left\{\alpha_{j l}\right\}$ respectively). Also, since $x \in$ Aut $\Gamma_{1}^{*}$, for any $k \notin\left\{i_{1}, \ldots, i_{t}\right\}$ and any $j, \phi_{k}$ is joined to all or no vertices of $\Delta_{j}^{\prime}$. From these facts we see that Aut $\Gamma$ contains a subgroup $H \cong S_{n+1}$ having orbits $\Delta_{1}^{\prime}, \ldots, \Delta_{t}^{\prime}$ and fixing $\phi_{k}$ for $k \notin\left\{i_{1}, \ldots, i_{t}\right\}$. Hence $\Gamma \in \mathscr{E}_{n+1}$.

THEOREM 1.4. Let $\Gamma \in \mathscr{E}_{n}$ have vertex set $\Delta_{1} \cup \cdots \cup \Delta_{t} \cup\left\{\phi_{1}, \ldots, \phi_{r}\right\}$ as above and let $\Gamma^{*}, \Gamma_{0}^{*}, \Gamma_{1}^{*}$ be the graphs corresponding to $\Gamma$. Suppose that Aut $\Gamma \cong S_{n}$. Then

(a) Aut $\Gamma^{*}=1$;

(b) $\Gamma_{0}^{*}$ is connected (by the black edges);

(c) Aut $\Gamma_{1}^{*}$ contains no automorphisms $\left(\delta_{1} \phi_{i_{1}}\right) \cdots\left(\delta_{t} \phi_{i_{t}}\right)$, with $i_{1}, \ldots, i_{t}$ distinct, such that

(i) $\phi_{i_{j}}$ is joined to $\delta_{j}$ in $\Gamma^{*}$ if and only if $\delta_{j}$ is black $(j=1, \ldots, t)$,

(ii) for any distinct $k$, l the subgraph $\left\{\delta_{k}, \delta_{l}, \phi_{i_{k}}, \phi_{i_{1}}\right\}$ of $\Gamma^{*}$ lies in $\mathscr{C}_{n}$.

Proof. (a) Suppose that $h \in$ Aut $\Gamma^{*}$ with $h \neq 1$. Define a permutation $g$ on $V \Gamma$ as follows

(1) if $\delta_{i} h=\delta_{j}$ put $\alpha_{i k} g=\alpha_{j k}(k=1, \ldots, n)$,

(2) for $i=1, \ldots, r$ put $\phi_{i} g=\phi_{i} h$.

It is easy to check that $g \in$ Aut $\Gamma$, which contradicts the fact that since Aut $\Gamma \cong S_{n}$, Aut $\Gamma$ has orbits $\Delta_{1}, \ldots, \Delta_{t}$ and fixes each $\phi_{j}$. 
(b) Suppose that $\Gamma_{0}^{*}$ is disconnected and let $\left\{\delta_{i_{1}}, \ldots, \delta_{i_{u}}\right\}(u<t)$ be a connected component of $\Gamma_{0}^{*}$; write $\Delta=\bigcup_{j=1}^{u} \Delta_{i_{j}}$. Then for any $\beta \in V \Gamma \backslash \Delta$ and any $j \in\{1, \ldots, u\}, \beta$ is joined to all or no vertices in $\Delta_{i,}$. Hence Aut $\Gamma$ contains a subgroup $H \cong S_{n}$ with orbits $\Delta_{i_{1}}, \ldots, \Delta_{i_{u}}$ and fixing every vertex in $V \Gamma \backslash \Delta$. Since $u<t$ it is clear that $H \neq$ Aut $\Gamma$, contradicting the fact that Aut $\Gamma \cong S_{n}$. Thus $\Gamma_{0}^{*}$ is connected.

(c) This follows directly from Lemma 1.3.

It seems likely that a general converse of Theorem 1.4 holds; since we have only been able to prove this when $t \leqslant 4$, we state the general case as a conjecture.

CONJECTURE 1.5. Let $n, t$ be positive integers with $n>t$. Let $\Gamma^{*}$ be a graph on vertex set $\left\{\delta_{1}, \ldots, \delta_{t}, \phi_{1}, \ldots, \phi_{r}\right\}$ with 3 vertex-colours (white and black among the $\delta_{i}$, red for the $\left.\phi_{i}\right)$ and 5 edge-colours $\left(0,1, n-1, n\right.$ for edges between the $\delta_{i}$, black for any other edges). Let $\Gamma_{0}^{*}$ and $\Gamma_{1}^{*}$ be the graphs defined from $\Gamma^{*}$ as above and suppose that these satisfy conditions (a), (b) and (c) of Theorem 1.4. Then if $\Gamma$ is the graph on $t n+r$ vertices corresponding as above to $\Gamma^{*}$, we have Aut $\Gamma \cong S_{n}$.

In the next sections we prove Conjecture 1.5 for $1 \leqslant t \leqslant 4$ and give some illustrations of its use in describing graphs $\Gamma$ with Aut $\Gamma \cong S_{n}$. It should be noted that we have introduced the condition $n>t$ in Conjecture 1.5 solely for convenience in the proofs in $\$ 2$, and that it seems likely that the conjecture is true for any values of $n$ and $t$ with $n \geqslant 3$.

\section{Proofs of Conjecture 1.5 for $1 \leqslant t \leqslant 4$}

The case $t=1$. We prove Conjecture 1.5 for $t=1$. Let $\Gamma^{*}$ be a graph on $\left\{\delta_{1}, \phi_{1}, \ldots, \phi_{r}\right\}$ coloured as in 1.5 . We assume first that $\delta_{1}$ is black. Writing $H=$ Aut $\Gamma_{1}^{*}$, condition (a) of Theorem 1.4 means that

(1) $H_{\delta_{1}}=1$, condition (b) is vacuously satisfied and condition (c) means that

(2) $H$ contains no automorphism $\left(\delta_{1} \phi_{i_{1}}\right)$ with $\delta_{1}$ joined to $\phi_{i_{1}}$.

Suppose then that (1) and (2) hold and let $\Gamma$ be the corresponding graph on $n+r$ vertices $\Delta_{1} \cup\left\{\phi_{1}, \ldots, \phi_{r}\right\}$, where $\Delta_{1}=\left\{\alpha_{11}, \ldots, \alpha_{1 n}\right\}$ is $K_{n}$ since $\delta_{1}$ is black. Write $G=$ Aut $\Gamma$. We show that $G \cong S_{n}$.

Since $H_{\delta_{1}}=1$ it is clear that $G_{\left\{\Delta_{1}\right\}}$ fixes $V \Gamma \backslash \Delta_{1}$ pointwise; thus $G_{\left\{\Delta_{1}\right\}} \cong S_{n}$. Suppose that there exists $g \in G \backslash G_{\left\{\Delta_{1}\right\}}$. Then $G_{\left\{\Delta_{1} g\right\}}$ fixes $V \Gamma \backslash \Delta_{1} g$ pointwise and $G_{\left(\Delta_{1} g\right)} \cong S_{n}$, so if $\Delta_{1} \cap \Delta_{1} g=\varnothing$ then $H_{\delta_{1}}$ has a subgroup isomorphic to $S_{n}$, contradicting (1). Hence $\Delta_{1} \cap \Delta_{1} g \neq \varnothing$. Write $\Sigma=\Delta_{1} \cup \Delta_{1} g$. It is easy to see that $G_{\{\Sigma\}}=\left\langle G_{\left\{\Delta_{1}\right\}}, G_{\left\{\Delta_{1} g\right\}}\right\rangle \cong \operatorname{Sym}(\Sigma)$ and that $G_{\{\Sigma\}}$ fixes $V \Gamma \backslash \Sigma$ pointwise. 
Consequently $\Sigma$ is a complete subgraph of $\Gamma$ and if we choose $\phi_{i_{1}} \in \Sigma \backslash \Delta_{1}$ then $\left(\alpha_{11} \phi_{i_{1}}\right) \in$ Aut $\Gamma$. This forces $\left(\delta_{1} \phi_{i_{1}}\right) \in$ Aut $\Gamma_{1}^{*}$, contradicting (2), as $\phi_{i_{1}}$ is joined to $\delta_{1}$.

The case where $\delta_{1}$ is white follows from the above argument by considering the complement of the corresponding graph $\Gamma$. Hence Conjecture 1.5 is proved for $t=1$.

Descriptions of the graphs characterized by this result can be found in the Appendix.

The cases $2 \leqslant t \leqslant 4$. We prove Conjecture 1.5 just for $t=4$, as the cases $t=2$ and $t=3$ are similar and easier. In the proof we shall need, for $2 \leqslant u \leqslant 4$ and $n>u$, a description of all coloured graphs $\Gamma^{*}$ on $\left\{\delta_{1}, \ldots, \delta_{u}\right\}$ which give rise as in $\S 1$ to vertex-transitive graphs $\Gamma$ on $u n$ vertices. We call $\Gamma^{*}$ vertex-monochrome if all the vertices $\delta_{i}$ have the same colour. are $u=2$. The only graphs $\Gamma^{*}$ on $\left\{\delta_{1}, \delta_{2}\right\}$ which give rise to a transitive graph $\Gamma$

$$
\delta_{1} \curvearrowleft \quad \circ \delta_{2} \quad \text { and } \quad \delta_{1} \bullet \quad a \quad \delta_{2}
$$

where $a$ is $0,1, n-1$ or $n$ (that is, $\Gamma^{*}$ is any vertex-monochrome graph on $\left.\left\{\delta_{1}, \delta_{2}\right\}\right)$.

$u=3$. Suppose that $\Gamma^{*}$ on $\left\{\delta_{1}, \delta_{2}, \delta_{3}\right\}$ gives rise to a transitive graph $\Gamma$. If $\Gamma^{*}$ is vertex-monochrome then by the regularity of $\Gamma$ it must be one of the following graphs:
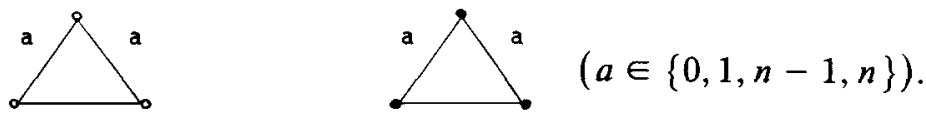

There are no further such graphs $\Gamma^{*}$. For suppose that $\Gamma^{*}$ is not vertex-monochrome. Then we may take $\Gamma^{*}$ to be

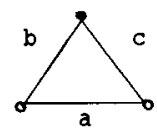

where $a, b, c \in\{0,1, n-1, n\}$. Since $\Gamma$ is regular we have

$$
a+b=a+c=b+c+n-1
$$

so that $b=c$ and $a=c+n-1$. Thus $c$ is 0 or 1 , which forces $\Delta_{3}$ to be the unique subgraph $K_{n}$ of $\Gamma$, contradicting the transitivity of $\Gamma$.

$u=4$. Suppose that $\Gamma^{*}$ on $\left\{\delta_{1}, \delta_{2}, \delta_{3}, \delta_{4}\right\}$ gives rise to a transitive graph $\Gamma$. Let $a_{i j} \in\{0,1, n-1, n\}$ be the colour of the edge joining $\delta_{i}$ and $\delta_{j}$. If $\Gamma^{*}$ is vertex-monochrome then since $\Gamma$ is regular of valency $b$, say, we have

$$
a_{12}+a_{13}+a_{14}=a_{12}+a_{23}+a_{24}=a_{13}+a_{23}+a_{34}=a_{14}+a_{24}+a_{34}=b \text {. }
$$


This gives $a_{14}=a_{23}, a_{13}=a_{24}, a_{12}=a_{34}$, so Aut $\Gamma^{*}$ contains the subgroup $V_{4}=\left\langle\left(\delta_{1} \delta_{2}\right)\left(\delta_{3} \delta_{4}\right),\left(\delta_{1} \delta_{3}\right)\left(\delta_{2} \delta_{4}\right)\right\rangle$. If $\delta_{1}$ is black and $\delta_{2}, \delta_{3}, \delta_{4}$ are white then the regularity of $\Gamma$ gives $2\left(a_{23}-a_{14}\right)=n-1$ which is not possible since $n>u=4$. And if $\delta_{1}, \delta_{2}$ are black and $\delta_{3}, \delta_{4}$ are white then it is easy to see that any vertex in $\Delta_{1} \cup \Delta_{2}$ is contained in more subgraphs $K_{n}$ of $\Gamma$ than any vertex in $\Delta_{3} \cup \Delta_{4}$, contradicting the transitivity of $\Gamma$.

We summarise these results in a lemma:

LeMMA 2.1. Let $\Gamma^{*}$ be a coloured graph on $\left\{\delta_{1}, \ldots, \delta_{u}\right\}(u \leqslant 4)$ which gives rise as in $\$ 1$ to a transitive graph $\Gamma$ on un vertices $(n>u)$. Then $\Gamma^{*}$ is vertex-monochrome and Aut $\Gamma^{*}$ contains a subgroup $S$, where $S=S_{2}$ if $u=2, S=S_{3}$ if $u=3$ and $S=V_{4}$ if $u=4$.

Proof of Conjecture 1.5 For $t=4$. Let $\Gamma^{*}$ be a graph on $\left\{\delta_{1}, \delta_{2}, \delta_{3}, \delta_{4}\right.$, $\left.\phi_{1}, \ldots, \phi_{r}\right\}$ coloured as in 1.5 and suppose that (a), (b) and (c) of Theorem 1.4 hold. Let $n$ be an integer with $n>t=4$ and let $\Gamma$ be the corresponding graph on $4 n+r$ vertices $\Delta_{1} \cup \Delta_{2} \cup \Delta_{3} \cup \Delta_{4} \cup\left\{\phi_{1}, \ldots, \phi_{r}\right\}$ (where $\Delta_{i}=\left\{\alpha_{i 1}, \ldots, \alpha_{i n}\right\}$ for $i=1, \ldots, 4)$. Write $\Delta=\Delta_{1} \cup \Delta_{2} \cup \Delta_{3} \cup \Delta_{4}$ and $\Phi=\left\{\phi_{1}, \ldots, \phi_{r}\right\}$, and let $\Delta^{*}$ be the subgraph of $\Gamma^{*}$ on $\left\{\delta_{1}, \delta_{2}, \delta_{3}, \delta_{4}\right\}$. Also write $\phi_{i} \sim \delta_{j}$ if $\phi_{i}$ is joined to $\delta_{j}$ in $\Gamma^{*}$. Put $G=$ Aut $\Gamma$. By the construction of $\Gamma$ from $\Gamma^{*}$ (explained in $\$ 1$ ) it is clear that $G$ has a unique subgroup $H \cong S_{n}$ having orbits $\Delta_{1}, \Delta_{2}, \Delta_{3}, \Delta_{4}$, fixing each $\phi_{i}$, and such that $H_{\alpha_{i j}}=H_{\alpha_{i k}}$ for all $i, j, k$. We aim to show that $G_{\{\Delta\}}=H$, which we establish in the following two lemmas.

Lemma 2.2. If $g \in G_{\{\Delta\}} \backslash H$ then $\Delta_{i} g \neq \Delta_{i}$ for some $i \in\{1,2,3,4\}$.

Proof. Suppose that $\Delta_{i} g=\Delta_{i}$ for all $i$. Then clearly $g^{\Phi} 1^{\Delta^{*}} \in$ Aut $\Gamma^{*}$, so $g^{\Phi}=1$ by (a) of 1.4. Now $H^{\Delta_{1}} \cong S_{n}$, so $g^{\Delta_{1}}=h^{\Delta_{1}}$ for some $h \in H$. Then $g^{-1} h$ fixes $\Delta_{1} \cup \Phi$ pointwise and $g^{-1} h \neq 1$ as $g \notin H$. Hence the sets

$$
\Delta^{\prime}=\bigcup\left\{\Delta_{i} \mid\left(g^{-1} h\right)^{\Delta_{i}}=1\right\}, \quad \Delta^{\prime \prime}=\bigcup\left\{\Delta_{i} \mid\left(g^{-1} h\right)^{\Delta_{1}} \neq 1\right\}
$$

are both nonempty. Let $K=\left\langle\left(g^{-1} h\right)^{x} \mid x \in H\right\rangle$. Then $K^{\Delta^{\prime}}=1$ and for $\Delta_{i} \subseteq \Delta^{\prime \prime}$ we have $K^{\Delta_{i}} \geqslant \operatorname{Alt}\left(\Delta_{i}\right)$ since $K^{\Delta_{i}} \triangleleft \operatorname{Sym}\left(\Delta_{i}\right)$. Hence for any $\Delta_{i} \subseteq \Delta^{\prime \prime}$ and any $\alpha_{j k} \in \Delta^{\prime}, \alpha_{j k}$ is joined to all or no vertices of $\Delta_{i}$. Thus in $\Gamma^{*}$, any edge between a vertex of $\left\{\delta_{i} \mid \Delta_{i} \subseteq \Delta^{\prime}\right\}$ and a vertex of $\left\{\delta_{i} \mid \Delta_{i} \subseteq \Delta^{\prime \prime}\right\}$ must be coloured 0 or $n$. This forces $\left\{\delta_{i} \mid \Delta_{i} \subseteq \Delta^{\prime}\right\}$ to be a union of connected components of the graph $\Gamma_{0}^{*}$, contradicting (b) of 1.4 . 
LEMMA 2.3. We have $G_{(\Delta)}=H$.

Proof. Suppose false and pick $g \in G_{\{\Delta\}} \backslash H$. By Lemma 2.2 we have $\Delta_{i} g \neq \Delta_{i}$ for some $i$, so if $L=\langle H, g\rangle$ then $L$ has at most 3 orbits on $\Delta$. We prove the lemma by obtaining a contradiction to the fact that Aut $\Gamma^{*}=1$. There are several cases, depending on the number of orbits of $L$ on $\Delta$.

Case 1. $L$ is transitive on $\Delta$. For any $i, j \in\{1,2,3,4\}$ write $\Delta_{i} \rightarrow \Delta_{j}$ if there exist $\alpha_{i k} \in \Delta_{i}, \quad \alpha_{j l} \in \Delta_{j}$ with $\alpha_{i k} g=\alpha_{j l}$. For distinct $i_{1}, \ldots, i_{u} \in\{1,2,3,4\}$ $(1 \leqslant u \leqslant 4)$ write $\left[\Delta_{i_{1}} \cdots \Delta_{i_{u}}\right]$ to mean that $\Delta_{i_{1}} \rightarrow \Delta_{i_{2}}, \Delta_{i_{2}} \rightarrow \Delta_{i_{3}}, \ldots, \Delta_{i_{u}} \rightarrow \Delta_{i_{1}}$.

Now $H$ has orbits $\Delta_{1}, \Delta_{2}, \Delta_{3}, \Delta_{4}$ on $\Delta$ and $L=\langle H, g\rangle$ is transitive on $\Delta$; it is not hard to see from this that we may assume that one of the following holds

(i) $\left[\Delta_{1} \Delta_{2} \Delta_{3} \Delta_{4}\right]$;

(ii) $\left[\Delta_{1} \Delta_{2}\right],\left[\Delta_{1} \Delta_{3}\right]$ and $\left[\Delta_{1} \Delta_{4}\right]$;

(iii) $\left[\Delta_{1} \Delta_{2}\right],\left[\Delta_{1} \Delta_{3}\right]$ and $\left[\Delta_{2} \Delta_{4}\right]$;

(iv) $\left[\Delta_{1} \Delta_{2} \Delta_{3}\right]$ and $\left[\Delta_{1} \Delta_{4}\right]$;

(v) $\left[\Delta_{1} \Delta_{2} \Delta_{3}\right]$ and $\left[\Delta_{1} \Delta_{2} \Delta_{4}\right]$.

Suppose that (i) holds. Then $\alpha_{1 i_{1}} g=\alpha_{2 i_{2}}, \alpha_{2 j_{2}} g=\alpha_{3 j_{3}}, \alpha_{3 k_{3}} g=\alpha_{4 k_{4}}, \alpha_{4 l_{4}} g=$ $\alpha_{1 i_{1}}$ for some $i_{1}, i_{2}$, etc. Choose $\phi_{a} \in \Phi$. If $\phi_{a}$ is joined to $\alpha_{1 i_{1}}$ then $\phi_{a} g$ is joined to $\alpha_{2 i}$, hence to every vertex in $\Delta_{2}$, so $\phi_{a} g^{2}$ is joined to $\alpha_{3 j_{3}}$. Thus $\phi_{a} \sim \delta_{1} \Rightarrow$ $\phi_{a} g^{2} \sim \delta_{3}$. In this way we see that $\phi_{a} \sim \delta_{1} \Rightarrow \phi_{a} g^{2} \sim \delta_{3}, \phi_{a} \sim \delta_{2} \Rightarrow \phi_{a} g^{2} \sim \delta_{4}$, $\phi_{a} \sim \delta_{3} \Rightarrow \phi_{a} g^{2} \sim \delta_{1}$ and $\phi_{a} \sim \delta_{4} \Rightarrow \phi_{a} g^{2} \sim \delta_{2}$. Also by Lemma 2.1 we have $\left(\delta_{1} \delta_{3}\right)\left(\delta_{2} \delta_{4}\right) \in$ Aut $\Delta^{*}$. It follows that $\left(g^{2}\right)^{\Phi}\left(\delta_{1} \delta_{3}\right)\left(\delta_{2} \delta_{4}\right) \in$ Aut $\Gamma^{*}$, contradicting the fact that Aut $\Gamma^{*}=1$.

If (ii) holds then for any $a \in\{1, \ldots, r\}$ we have $\phi_{a} \sim \delta_{2} \Leftrightarrow \phi_{a} g \sim \delta_{1} \Leftrightarrow$ $\phi_{a} \sim \delta_{3} \Leftrightarrow \phi_{a} \sim \delta_{4}$. Hence any permutation of $\left\{\delta_{2}, \delta_{3}, \delta_{4}\right\}$ fixing $\delta_{1}$ and each $\phi_{a}$ will be an automorphism of $\Gamma^{*}$ providing it is an automorphism of the subgraph $\Delta^{*}$. By Lemma 2.1 we can take $\Delta^{*}$ to be

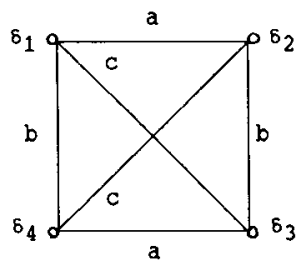

where $a, b, c \in\{0,1, n-1, n\}$. If $a=b$ then $\left(\delta_{2} \delta_{4}\right) \in$ Aut $\Gamma^{*}$, if $b=c$ then $\left(\delta_{3} \delta_{4}\right) \in$ Aut $\Gamma^{*}$ and if $a=c$ then $\left(\delta_{2} \delta_{3}\right) \in$ Aut $\Gamma^{*}$, all of which are contradictions. Hence, $a, b, c$ are distinct and we may assume that either $a=0, b=1$, $c \geqslant n-1$ or $a \leqslant 1, b=n-1, c=n$. Write $m\left(\alpha_{i j}, \alpha_{k l}\right)$ for the number of mutual adjacencies of $\alpha_{i j}$ and $\alpha_{k l}$ in the subgraph $\Delta$ of $\Gamma$. Then for any $i, j, k, l$ 
we have $m\left(\alpha_{1 i}, \alpha_{1 j}\right) \geqslant n-2$ and $m\left(\alpha_{2 k}, \alpha_{3 l}\right) \leqslant 2$. However, by assumption (we are in case (ii)) there exist $i, j, k, l$ such that $\alpha_{1 i} g=\alpha_{2 k}, \alpha_{1 j} g=\alpha_{3 l}$, which forces $m\left(\alpha_{1 i}, \alpha_{1 j}\right)=m\left(\alpha_{2 k}, \alpha_{3 l}\right)$; hence $n-2 \leqslant 2$ or $n \leqslant 4$, contradicting the fact that $n>t=4$.

In case (iii) we have $\phi_{a} \sim \delta_{1} \Leftrightarrow \phi_{a} g \sim \delta_{2} \Leftrightarrow \phi_{a} \sim \delta_{4}$ and $\phi_{a} \sim \delta_{2} \Leftrightarrow \phi_{a} g \sim \delta_{1}$ $\Leftrightarrow \phi_{a} \sim \delta_{3}$. Hence $\left(\delta_{1} \delta_{4}\right)\left(\delta_{2} \delta_{3}\right) \in$ Aut $\Gamma^{*}$ which is a contradiction.

In case (iv) we see similarly that $\left(\delta_{1} \delta_{2}\right)\left(\delta_{3} \delta_{4}\right) \in$ Aut $\Gamma^{*}$, again a contradiction.

Finally, suppose that (v) holds. Then $\phi_{a} \sim \delta_{3} \Leftrightarrow \phi_{a} g \sim \delta_{1} \Leftrightarrow \phi_{a} \sim \delta_{4}$ so $\left(\delta_{3} \delta_{4}\right) \in$ Aut $\Gamma^{*}$ if $b=c$ in the subgraph $\Delta^{*}$. Thus $b \neq c$. Suppose first that $c \leqslant 1$. Then $m\left(\alpha_{3 i}, \alpha_{4 j}\right) \leqslant 2$ for any $i, j$, so by application of $g^{-1}$ we see that $m\left(\alpha_{1 k}, \alpha_{1 l}\right) \leqslant 2$ for any distinct $k, l$. This forces $a \leqslant 1$ and $b \leqslant 1$. Since $b \neq c$ we may take $b=1, c=0$; as $\Gamma_{0}^{*}$ is connected we have $a=1$ and $\Delta^{*}$ is

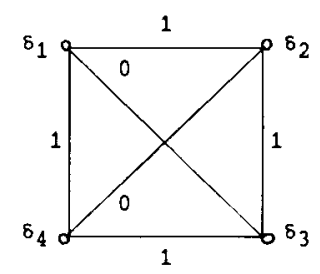

Thus the subgraph $\Delta$ of $\Gamma$ consists of $n$ disjoint squares. Now $\alpha_{1 i} g=\alpha_{2 j}$ for some $i, j$. Since $\alpha_{3 i}$ is the unique vertex of $\Delta$ opposite to $\alpha_{1 i}$ in the square containing $\alpha_{1 i}$ and $\alpha_{4 j}$ is similarly opposite to $\alpha_{2 j}$, we must have $\alpha_{3 i} g=\alpha_{4 j}$. In this way we see that $\left[\Delta_{3} \Delta_{4} \Delta_{1}\right]$ and $\left[\Delta_{3} \Delta_{4} \Delta_{2}\right]$ also hold. The usual argument now shows that $\phi_{a} \sim \delta_{1} \Leftrightarrow \phi_{a} \sim \delta_{2} \Leftrightarrow \phi_{a} \sim \delta_{3} \Leftrightarrow \phi_{a} \sim \delta_{4}$ so that $V_{4} \leqslant$ Aut $\Gamma^{*}$, which is a contradiction. Similar arguments yield a contradiction if $c \geqslant n-1$.

We have now dealt completely with Case 1.

Case 2. $L$ has orbits $\Delta_{1} \cup \Delta_{2} \cup \Delta_{3}$ and $\Delta_{4}$ on $\Delta$. In this case we may assume that either (i) $\left[\Delta_{1} \Delta_{2} \Delta_{3}\right]$, or (ii) $\left[\Delta_{1} \Delta_{2}\right]$ and $\left[\Delta_{1} \Delta_{3}\right]$ holds. Using Lemma 2.2 for $u=3$ and the fact that each vertex in $\Delta_{1} \cup \Delta_{2} \cup \Delta_{3}$ has the same valency, we see that the subgraph $\Delta^{*}$ can be taken to be one of
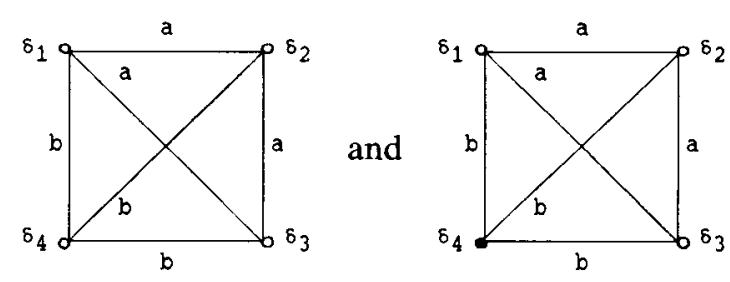
for some $a, b \in\{0,1, n-1, n\}$. If (i) holds then $g^{\Phi}\left(\delta_{1} \delta_{2} \delta_{3}\right) \in$ Aut $\Gamma^{*}$, while in case (ii) we have $\phi_{a} \sim \delta_{2} \Leftrightarrow \phi_{a} g \sim \delta_{1} \Leftrightarrow \phi_{a} \sim \delta_{3}$ so that $\left(\delta_{2} \delta_{3}\right) \in$ Aut $\Gamma^{*}$. These contradictions deal with Case 2.

Case 3. $L$ has orbits $\Delta_{1} \cup \Delta_{2}$ and $\Delta_{3} \cup \Delta_{4}$ or $\Delta_{1} \cup \Delta_{2}, \Delta_{3}$ and $\Delta_{4}$ on $\Delta$. Then either (i) $\left[\Delta_{1} \Delta_{2}\right]$ and $\left[\Delta_{3} \Delta_{4}\right]$, or (ii) $\left[\Delta_{1} \Delta_{2}\right],\left[\Delta_{3}\right]$ and $\left[\Delta_{4}\right]$, holds. In case (i) we have $g^{\Phi}\left(\delta_{1} \delta_{2}\right)\left(\delta_{3} \delta_{4}\right) \in$ Aut $\Gamma^{*}$ and in case (ii), $g^{\Phi}\left(\delta_{1} \delta_{2}\right) \in$ Aut $\Gamma^{*}$, neither of which can be so.

This completes the proof of Lemma 2.3.

To finish the proof of Conjecture 1.5 for $t=4$ it remains to show that $G=G_{\{\Delta\}}$. Suppose then that there exists $g \in G \backslash G_{\{\Delta\}}$. Put $M=\left\langle G_{\{\Delta\}}, G_{\{\Delta\}}\right\rangle$ $=\left\langle H, H^{g}\right\rangle$ and let $\Psi_{1}, \ldots, \Psi_{s}$ be the orbits of $M$ on $\Delta \cup \Delta g$. For each $i$ let $X_{i}=\left\{j \mid \Delta_{j} \subseteq \Psi_{i}\right\}$.

LEMMA 2.4. If $X_{i} \neq \varnothing$ then there is a block system $\mathscr{B}_{i}$ for $M^{\Psi_{i}}$ (possibly with blocks of size 1$)$, one of whose blocks $B_{i}$ is contained in $\left\{\alpha_{j 1} \mid j \in X_{i}\right\}$, and such that $M^{\mathscr{S}_{i}} \geqslant \operatorname{Alt}\left(\mathscr{B}_{i}\right)$.

Proor. Pick $k \in X_{i}$, so that $\Delta_{k} \subseteq \Psi_{i}$. The lemma is certainly true if $\Delta_{k}=\Psi_{i}$ (for then we take $\mathscr{B}_{i}=\Psi_{i}$, that is, $\mathscr{B}_{i}$ to be the set of blocks of size 1 ); hence we may assume that $\Delta_{k} \subset \Psi_{i}$. Let $\mathscr{B}_{i}$ be a block system for $M^{\Psi_{i}}$ such that $\left|\mathscr{B}_{i}\right|>1$ and $\mathscr{B}_{i}$ contains blocks of maximum possible size. Then $M^{\mathscr{B}}$ is primitive. Let $B_{i}$ be the block of $\mathscr{B}_{i}$ containing $\alpha_{k 1}$. Certainly either $\Delta_{k} \subseteq B_{i}$ or $\Delta_{k} \cap B_{i}=\left\{\alpha_{k 1}\right\}$; we show that the latter must hold. Suppose then that $\Delta_{k} \subseteq B_{i}$. From the action of $H$ we have

(1) $B_{i} \cap \Delta$ is a union of $H$-orbits $\Delta_{j}$.

Next we show that

(2) $B_{i} \cap \Delta g$ is a union of $H^{g}$-orbits $\Delta_{j} g$.

We prove this as follows: if $\left|\Delta_{k} \cap \Delta_{l} g\right| \leqslant 1$ for all $l$ then since $n>t$ there exists $\alpha_{k m} \in \Delta_{k} \backslash \Delta g$, so that $H^{g}$ fixes $\alpha_{k m}$. Now $\Delta_{k} \subset \Psi_{i}$ so we can find $l$ such that $\Delta_{k} \cap \Delta_{l} g \neq \varnothing$. Also $\Delta_{k} \subseteq B_{i}$, so $B_{i} \cap \Delta_{l} g \neq \varnothing$. Since $H^{g}$ fixes $\alpha_{k m}$ this forces $\Delta_{l} g \subseteq B_{i}$. The action of $H^{g}$ now gives (2). If $\left|\Delta_{k} \cap \Delta_{l} g\right| \geqslant 2$ for some $l$ then $\Delta_{1} g \subseteq B_{i}$ again, from which (2) follows as before. Hence (2) is established. Now $M=\left\langle H, H^{g}\right\rangle$ and $\Psi_{i}$ is a union of sets $\Delta_{j}$ and $\Delta_{j} g$ on which $M$ is transitive. It follows from (1) and (2) that $B_{i}=\Psi_{i}$, contradicting the fact that $\left|\mathscr{B}_{i}\right|>1$.

Thus we have shown that $\Delta_{k} \cap B_{i}=\left\{\alpha_{k 1}\right\}$. Since $H_{\alpha_{k 1}}=H_{\alpha_{11}}$ for all $j$, it follows that $B_{i} \subseteq\left\{\alpha_{j 1} \mid j \in X_{i}\right\}$. Finally, $M^{\mathscr{B}_{i}}$ is primitive and contains the subgroup $H^{\mathscr{T}_{i}} \cong S_{n}$, so $M^{\mathscr{S}_{i}}$ contains an element of degree at most 8 . From this it follows without much difficulty that $M^{\mathscr{B}_{i}} \geqslant \operatorname{Alt}\left(\mathscr{B}_{i}\right)$ (see for instance the papers of W. A. Manning referred to at the end of $\$ 15$ of [4]). 
If $X_{i}=\varnothing$ then $\Psi_{i}=\Delta_{j} g$ for some $j$ and $M^{\Psi_{i}}=\operatorname{Sym}\left(\Psi_{i}\right)$. Put $\mathscr{B}_{i}=\Psi_{i}$ in this case (that is, let $\mathscr{B}_{i}$ be the set of blocks of size 1). Choose notation so that $X_{i} \neq \varnothing$ for $i=1, \ldots, s_{0}$ and $X_{i}=\varnothing$ for $i=s_{0}+1, \ldots, s$. For $i \in\left\{1, \ldots, s_{0}\right\}$ let $\mathscr{B}_{i}^{\prime}$ be the set of blocks of $\mathscr{B}_{i}$ contained in $\Delta$. Then $\left|\mathscr{B}_{i}^{\prime}\right|=r_{i} n$ for some positive integer $r_{i}$. Write $\mathscr{B}=\bigcup_{i=1}^{s} \mathscr{B}_{i}$.

LEMMA 2.5. The following hold:

(i) $s=s_{0}$;

(ii) $\left|\mathscr{B}_{j}\right|=\left|\mathscr{B}_{k}\right|$ for all $j, k \in\{1, \ldots, s\}$;

(iii) if $\left|\mathscr{B}_{1}\right|=b$ then $M \cong A_{b}$ or $M \cong S_{b}$ and $M$ acts similarly on each $\mathscr{B}_{j}$ $(j=1, \ldots, s)$.

Proof. If $K$ is the kernel of the action of $M$ on $\mathscr{B}$ then $K \leqslant G_{(\Delta\}}$, so $K=1$ since $G_{\{\Delta\}}=H$. Hence $M$ acts faithfully on $\mathscr{B}$. Write $N=M^{\prime}$. Then by Lemma 2.4, $N$ is a subdirect product of $\prod_{i=1}^{s} \operatorname{Alt}\left(\mathscr{B}_{i}\right)$ (that is, $N$ projects surjectively onto each factor). Since each $\operatorname{Alt}\left(\mathscr{B}_{i}\right)$ is simple, $N$ is isomorphic to a direct product of some of the groups $\operatorname{Alt}\left(\mathscr{B}_{i}\right)$ and if we choose $i_{0}$ such that $\left|\mathscr{B}_{i_{0}}\right|=$ $\max \left\{\left|\mathscr{B}_{i}\right|: i=1, \ldots, s\right\}$ then $N$ has a minimal normal subgroup $N_{0} \cong \operatorname{Alt}\left(\mathscr{B}_{i_{0}}\right)$. Now $g \notin G_{\{\Delta\}}$, so $H^{g} \neq H$ and so $M \nless G_{\{\Delta\}}$. Consequently $\left|\mathscr{B}_{i_{0}}\right|>n$. Hence if $X_{i}=\varnothing$ then $\left|\mathscr{B}_{i_{0}}\right|>\left|\mathscr{B}_{i}\right|$. Let

$$
J=\left\{j \mid N_{0}^{\mathscr{j}}=\operatorname{Alt}\left(\mathscr{B}_{j}\right)\right\} \quad \text { and } \quad \mathscr{B}_{0}=\bigcup_{j \in J} \mathscr{B}_{j} .
$$

Then $J \subseteq\left\{1, \ldots, s_{0}\right\},\left|\mathscr{B}_{j}\right|=\left|\mathscr{B}_{i_{0}}\right|$ for all $j \in J$ and $N_{0}$ fixes $\mathscr{B} \backslash \mathscr{B}_{0}$ pointwise.

Write $H_{0}=H^{\prime}$; then $H_{0} \cong A_{n}$ and $H_{0}^{\mathscr{S}_{0}} \leqslant N_{0}^{\mathscr{B}_{0}}$. It follows that $N_{0}$ acts similarly on all $\mathscr{B}_{j}(j \in J)$ (whether $\left|\mathscr{B}_{i_{0}}\right|=6$ or not), and hence that $N_{0}$ contains a nontrivial element $x$ fixing each $\mathscr{B}_{j}^{\prime}$ setwise $(J \in J)$. Then $x$ fixes $\mathscr{B} \backslash \mathscr{B}_{0}$ pointwise, so $x \in G_{\{\Delta\}}$ and so $x \in H$. This forces $J=\left\{1, \ldots, s_{0}\right\}$. If $s>s_{0}$ then $N$ has a subgroup $L \cong A_{n}$ fixing $\bigcup_{i=1}^{s_{0}} \mathscr{B}_{i}$ pointwise; clearly $L \leqslant G_{\{\Delta\}}$, which is not possible as $G_{\{\Delta\}}=H$. Thus $s=s_{0}, J=\{1, \ldots, s\}, N=N_{0}$ and the lemma follows.

LemmA 2.6. We have $\left|\mathscr{B}_{i}^{\prime}\right|=n$, that is, $r_{i}=1$ for all $i$.

Proof. By Lemma $2.5, M$ has a subgroup $N_{1}$ fixing eacyh $\mathscr{B}_{i}^{\prime}$ setwise and such that $N_{1}^{\mathscr{B}_{i}^{\prime}} \geqslant \operatorname{Alt}\left(\mathscr{B}_{i}^{\prime}\right)(i=1, \ldots, s)$. Clearly $N_{1} \leqslant G_{\{\Delta\}}$. Since $G_{\{\Delta\}}=H \cong S_{n}$ this forces $\left|\mathscr{B}_{i}^{\prime}\right|=n$, that is, $r_{i}=1$, for all $i$.

We can now complete the proof of Conjecture 1.5 for $t=4$. First note that from the proof of Lemma 2.5 , we have $M \nless G_{\{\Delta\}}$. Hence there exists $k$ such that 
$X_{k} \neq \varnothing$ and $\Psi_{k} \nsubseteq \Delta$ (equivalently $\mathscr{B}_{k}^{\prime} \neq \mathscr{B}_{k}$ ). By Lemma 2.6 we have $\left|\mathscr{B}_{k}\right|=$ $n+c$ where $c>0$ is the number of blocks in $\mathscr{B}_{k} \backslash \mathscr{B}_{k}^{\prime}$. Thus by Lemma 2.5, $X_{i} \neq \varnothing,\left|\mathscr{B}_{i}\right|=n+c$ and $M^{\mathscr{B}_{i}} \cong S_{n+c}(i=1, \ldots, s)$. Finally, choose $B_{1}^{\prime} \in$ $\mathscr{B}_{1} \backslash \mathscr{B}_{1}^{\prime}$. There exists $m \in M$ with $m^{\mathscr{P}_{i}}=\left(B_{1} B_{1}^{\prime}\right)$. By Lemma $2.5, M$ acts similarly on all $\mathscr{B}_{i}$, so $m^{\mathscr{B}_{i}}=\left(B_{i} B_{i}^{\prime}\right)$ for some $B_{i}^{\prime} \in \mathscr{B}_{i} \backslash \mathscr{B}_{i}^{\prime}(i=1, \ldots, s)$. Since the kernel of the action of $M$ on $\mathscr{B}$ is trivial, we have $m^{2}=1$. Hence $m=$ $\left(\alpha_{11} \phi_{i_{1}}\right)\left(\alpha_{21} \phi_{i_{2}}\right) \cdots\left(\alpha_{t 1} \phi_{i_{t}}\right)$ for some $\phi_{i_{i}} \in \Phi(j=1, \ldots, t)$. From this it follows that $\left(\delta_{1} \phi_{i_{1}}\right) \cdots\left(\delta_{i} \phi_{i_{i}}\right) \in$ Aut $\Gamma_{1}^{*}$ and that for any distinct $k, l$ the subgraph $\left\{\delta_{k}, \delta_{l}, \phi_{i_{k}}, \phi_{i_{l}}\right\}$ of $\Gamma^{*}$ lies in the set $\mathscr{C}_{n}$ of 12 graphs defined in $\S 1$. This contradicts (c) of Theorem 1.4.

This completes the proof of Conjecture 1.5 for $t=4$.

We summarise the results proved in this section:

THEOREM 2.7. Let $n, t$ be integers with $1 \leqslant t \leqslant 4$ and $n>t$, and let $\Gamma^{*}$ be a graph on $\left\{\delta_{1}, \ldots, \delta_{t}, \phi_{1}, \ldots, \phi_{r}\right\}$ coloured as described in Conjecture 1.5. Suppose that (a), (b) and (c) of Theorem 1.4 are satisfied. Then if $\Gamma$ is the corresponding graph on th $+r$ vertices, we have Aut $\Gamma \cong S_{n}$.

The results $1.2,1.4$ and 2.7 give a description of all graphs $\Gamma$ on $v$ vertices with Aut $\Gamma \cong S_{n}(n>6)$ and $v<\min \left\{5 n, \frac{1}{2} n(n-1)\right\}$. This description is illustrated below in the Appendix. For values of $n$ with $n \leqslant 6$ there are some extra possibilities which can easily be determined using the techniques of this paper.

Remarks. 1. The restriction $n>t$ in Theorem 2.7 is in fact unnecessary-it is not hard to show that the result is true for any $n, t$ with $1 \leqslant t \leqslant 4, n \geqslant 3$.

2. The obstacle to a general proof of Conjecture 1.5 seems to lie solely in proving Lemma 2.3 in the general case; the subsequent steps of the proof for $t=4$ do not depend on the value of $t$ and would remain largely unchanged in the general case.

3. The methods of this paper could be used to study graphs with automorphism group $S_{n}$ having some orbit sizes greater than $\frac{1}{2} n(n-1)$. For example, suppose that we only restrict all orbits to have size less than $n(n-1)(n-2) / 6$. Then for $n$ large enough, the proofs of Propositions 1.1 and 1.2 show that all orbits have size $1, n$ or $\frac{1}{2} n(n-1)$ (with the action of $S_{n}$ in the latter case being that on the set of pairs of points in an underlying set of size $n$ ). There are four possible subgraphs on an orbit of size $\frac{1}{2} n(n-1)$ : these are the complete graph $K_{\frac{1}{2} n(n-1)}$, the triangular graph $T_{n}$ and their complements. By introducing a suitable collection of colours to represent these subgraphs and the edges between them, we can proceed in similar fashion to $\$ 1$. 


\section{Appendix}

In this Appendix we give descriptions of some of the graphs characterized by Theorems 1.4 and 2.7. In particular we describe all graphs $\Gamma$ with Aut $\Gamma \cong S_{n}$ and $|V \Gamma| \leqslant 3 n$ (with $n>6$ ). The reader will have no difficulty in extending these descriptions to cover all graphs $\Gamma$ with Aut $\Gamma \cong S_{n}$ and

$$
|V \Gamma|<\min \left\{5 n, \frac{1}{2} n(n-1)\right\} \text {. }
$$

It is unfortunately necessary to introduce some fairly complicated notation for these descriptions, so we include a number of small examples for illustration.

Throughout this Appendix, $\Gamma$ denotes a graph on $v$ vertices. For any $n$, let

$$
\mathscr{F}_{v, n}=\left\{\Gamma \mid \text { Aut } \Gamma \cong S_{n}\right\} \text {. }
$$

For any $t, r, n$ with $n>t$ define

$$
\begin{array}{r}
\mathscr{G}_{t, r, n}=\left\{\Gamma \mid v=t n+r, \text { Aut } \Gamma \cong S_{n} \text { has } t \text { orbits of size } n\right. \text { and } \\
r \text { fixed points on } V \Gamma\} .
\end{array}
$$

Thus by Proposition 1.2, for $n>6$ and $v<\frac{1}{2} n(n-1)$, we have

$$
\mathscr{F}_{v, n}=\bigcup_{1 \leqslant j \leqslant t} \mathscr{G}_{j,(t-j) n+r, n}
$$

where $v=t n+r$ and $0 \leqslant r<n$. Thus to describe $\mathscr{F}_{v, n}$ we must describe the graphs in $\mathscr{G}_{t, r, n}$. This can be done for $t \leqslant 4$ using Theorems 1.4 and 2.7, and we now give such descriptions explicitly, starting with the simplest case $t=1$. For convenience, if $\mathscr{S}$ is a set of graphs on $v$ vertices, define

$$
\mathscr{S}^{*}=\{\{\Gamma, \bar{\Gamma}\} \mid \Gamma \in \mathscr{S}\},
$$

where $\bar{\Gamma}$ denotes the complement of $\Gamma$.

(A) The case $t=1$. We describe $\mathscr{G}_{1, r, n}$. Let $\mathscr{H}_{1, r}$ be the set of (uncoloured) graphs $\Gamma_{1}^{*}$ on $1+r$ vertices $\left\{\delta_{1}, \phi_{1}, \ldots, \phi_{r}\right\}$ such that $H=$ Aut $\Gamma_{1}^{*}$ satisfies

(1) $H_{\delta_{1}}=1$, and,

(2) $H$ contains no element $\left(\delta_{1} \phi_{i_{1}}\right)$ with $\delta_{1}$ joined to $\phi_{i_{1}}$.

Each graph $\Gamma_{1}^{*}$ in $\mathscr{H}_{1, r}$ corresponds as in Section 1 to a graph $\Gamma$ on $n+r$ vertices as follows: $\Gamma$ has vertex set $\Delta_{1} \cup\left\{\phi_{1}, \ldots, \phi_{r}\right\}$, the subgraph $\Delta_{1}$ is $K_{n}$, the subgraph $\left\{\phi_{1}, \ldots, \phi_{r}\right\}$ is as in $\Gamma_{1}^{*}$, and $\phi_{i}$ is joined to all or no vertices in $\Delta_{1}$ according as $\phi_{i}$ is or is not joined to $\delta_{1}$ in $\Gamma_{1}^{*}$. By Theorem 2.7 with $t=1$, we have Aut $\Gamma \cong S_{n}$, so that $\Gamma$ is in $\mathscr{G}_{1, r, n}$.

Now the graphs in $\mathscr{G}_{1, r, n}$ are unlabelled, so we choose a subset $\mathscr{H}_{1, r}^{0}$ of $\mathscr{H}_{1, r}$ containing exactly one member of each orbit of $\operatorname{Sym}\left\{\phi_{1}, \ldots, \phi_{r}\right\}$ on $\mathscr{H}_{1, r}$. Then $\mathscr{G}_{1, r, n}^{*}$ is in 1-1 correspondence, as described above, with $\mathscr{H}_{1, r}^{0}$. We write this as

$$
\mathscr{G}_{1, r, n}^{*} \leftrightarrow \mathscr{H}_{1, r}^{0} .
$$


In particular, for $r<n$ we have by (1), $\mathscr{F}_{n+r, n}^{*} \leftrightarrow \mathscr{H}_{1, r}^{0}$. This gives the results of [3].

EXAMPLE. We illustrate this with $r=2$ and $r=3$. Each of $\mathscr{H}_{1,2}^{0}$ and $\mathscr{H}_{1,3}^{0}$ consists of just one graph:
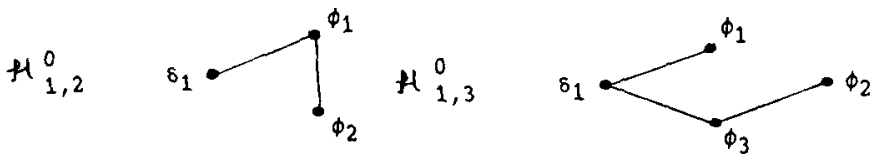

Thus $\mathscr{G}_{1,2, n}$ and $\mathscr{G}_{1,3, n}$ consist, respectively, of the graphs
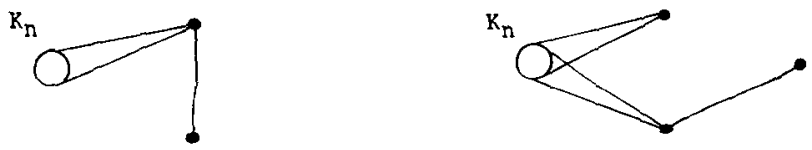

and their complements.

(B) The case $t=2$. We now describe $\mathscr{G}_{2, r, n}(n \geqslant 3)$. If $\Gamma \in \mathscr{G}_{2, r, n}$ and $\Gamma^{*}$ is the corresponding coloured graph on $\left\{\delta_{1}, \delta_{2}, \phi_{1}, \ldots, \phi_{r}\right\}$ then by (b) of Theorem 1.4 the subgraph of $\Gamma^{*}$ on $\delta_{1}, \delta_{2}$ is one of the following:
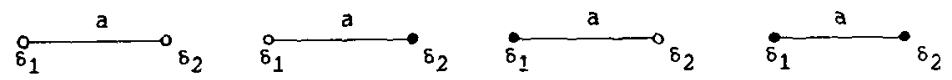

where $a$ is 1 or $n-1$. Let $\mathscr{H}_{2, r}$ be the set of (uncoloured) graphs $\Gamma_{1}^{*}$ on $\left\{\delta_{1}, \delta_{2}, \phi_{1}, \ldots, \phi_{r}\right\}$ such that $H=$ Aut $\Gamma_{1}^{*}$ satisfies

(1) $H_{\left\{\delta_{1} \delta_{2}\right\}}=1$, and

(2) $H$ contains no element $\left(\delta_{1} \phi_{i_{1}}\right)\left(\delta_{2} \phi_{i_{2}}\right)$ such that the subgraph $\left\{\delta_{1}, \delta_{2}, \phi_{i_{1}}, \phi_{i_{2}}\right\}$ is one of
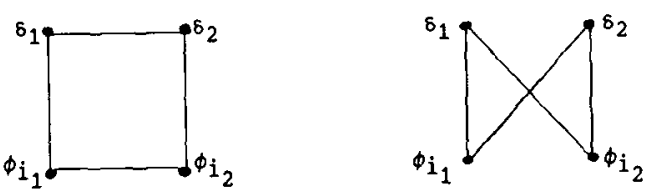

And let $\mathscr{H}_{2, r}^{\prime}$ be the set of graphs $\Gamma_{1}^{*}$ on $\left\{\delta_{1}, \delta_{2}, \phi_{1}, \ldots, \phi_{r}\right\}$ such that

(I) $H_{\delta_{1} \delta_{2}}=1$, and 
(II) $H$ contains no element $\left(\delta_{1} \phi_{i_{1}}\right)\left(\delta_{2} \phi_{i_{2}}\right)$ such that the subgraph $\left\{\delta_{1}, \delta_{2}, \phi_{i_{1}}, \phi_{i_{2}}\right\}$ is one of
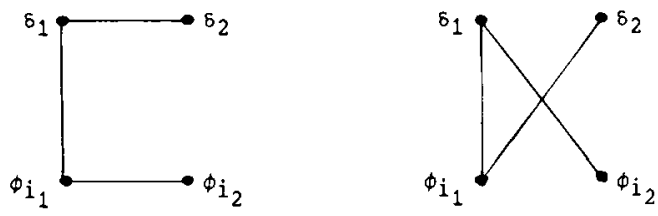

Choose a subset $\mathscr{H}_{2, r}^{0}$ of $\mathscr{H}_{2, r}$ containing exactly one member of each orbit of $\operatorname{Sym}\left\{\phi_{1}, \ldots, \phi_{r}\right\} \times \operatorname{Sym}\left\{\delta_{1}, \delta_{2}\right\}$ on $\mathscr{H}_{2, r} ;$ and choose a subset $\mathscr{H}_{2, r}^{\prime 0}$ containing exactly one member of each orbit of $\operatorname{Sym}\left\{\phi_{1}, \ldots, \phi_{r}\right\}$ on $\mathscr{H}_{2, r}^{\prime}$. Then each graph $\Gamma_{1}^{*}$ in $\mathscr{H}_{2, r}^{0}$ corresponds to a unique graph $\Gamma$ in $\mathscr{G}_{2, r, n}$ in which the subgraphs $\Delta_{1}$ and $\Delta_{2}$ are both $K_{n}$ (and $a=1$ if $\delta_{1}$ and $\delta_{2}$ are joined in $\Gamma_{1}^{*}, a=n-1$ if not). And each $\Gamma_{1}^{*}$ in $\mathscr{H}_{2, r}^{\prime 0}$ corresponds to a unique graph $\Gamma$ in $\mathscr{G}_{2, r, n}$ in which $\Delta_{1}$ is $K_{n}$ and $\Delta_{2}$ is $V_{n}$; the complement $\bar{\Gamma}$ then corresponds to the graph $\left(\Gamma_{1}^{*}\right)^{+}$, which is the image of the complement $\bar{\Gamma}_{1}^{*}$ under the transposition $\left(\delta_{1} \delta_{2}\right)$. Hence if we write

$$
\left(\mathscr{H}_{2, r}^{\prime 0}\right)^{+}=\left\{\left\{\Gamma_{1}^{*},\left(\Gamma_{1}^{*}\right)^{+}\right\} \mid \Gamma_{1}^{*} \in \mathscr{H}_{2, r}^{\prime 0}\right\}
$$

then we have

$$
\mathscr{G}_{2, r, n}^{*} \leftrightarrow \mathscr{H}_{2, r}^{0} \cup\left(\mathscr{H}_{2, r}^{\prime 0}\right)^{+} .
$$

Note that if $v=2 n+r<3 n$ and $n>6$, then by (1),

$$
\mathscr{F}_{v, n}=\mathscr{G}_{1, n+r, n} \cup \mathscr{G}_{2, r, n}
$$

so the description of $\mathscr{F}_{v, n}$ is given by (A) and the above.

EXAMPLE. We illustrate the above by producing the graphs in $\mathscr{G}_{2,0, n}$ and $\mathscr{G}_{2,1, n}$. Those in $\mathscr{G}_{2,0, n}$ correspond to the two coloured graphs

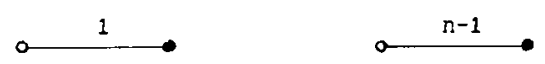

Thus $\mathscr{G}_{2,0, n}$ consists of the corona $K_{n} \circ K_{1}$ (which is $K_{n}$ with each vertex joined to just one further vertex) and its complement. This answers a question raised in [3, $\S 4]$. The graphs in $\mathscr{G}_{2,1, n}$ are those corresponding to the coloured graphs
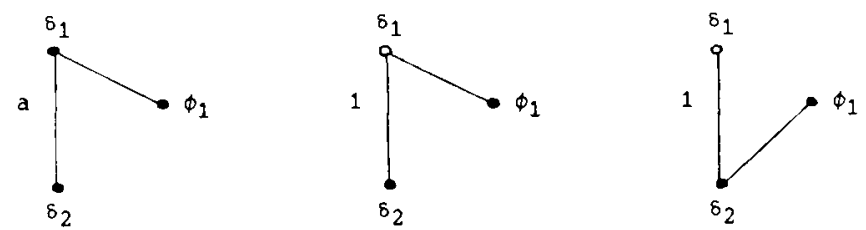

(where $a$ is 1 or $n-1$ ), together with their complements. 
Descriptions similar to, but rather more complicated than those given in (A) and (B), exist for $t=3$ and $t=4$. We leave these to the reader, and offer just one further illustration.

(C) We describe $\mathscr{G}_{3,0, n}(n \geqslant 4)$. By Theorems 1.4 and $2.7, \mathscr{G}_{3,0, n}^{*}$ is in $1-1$ correspondence with the following set $\mathscr{H}_{3,0}$ of coloured graphs:
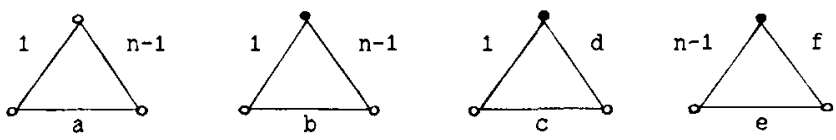

(any $b \in\{0,1, n-1, n\}, a, d, f \in\{0, n\}, c, e \in\{1, n-1\}$ ).

Hence for $n>6$,

$$
\begin{aligned}
\mathscr{F}_{3 n, n}^{*} & =\mathscr{G}_{1,2 n, n}^{*} \cup \mathscr{G}_{2, n, n}^{*} \cup \mathscr{G}_{3,0, n}^{*} \\
& \leftrightarrow \mathscr{H}_{1,2 n}^{0} \cup \mathscr{H}_{2, n}^{0} \cup\left(\mathscr{H}_{2, n}^{\prime 0}\right)^{+} \cup \mathscr{H}_{3,0} .
\end{aligned}
$$

\section{References}

[1] R. Frucht, 'Herstellung von Graphen mit vorgegebener abstrakten Gruppe', Compositio Math. 6 (1938), 239-250.

[2] A. Gewirtz and L. V. Quintas, 'Connected extremal edge graphs having symmetric automorphism group', Recent progress in combinatorics, ed. W. T. Tutte (Academic Press, New York, 1969).

[3] K. Heinrich and W. D. Wallis, 'Graphs with symmetric automorphism group', J. Graph Theory 2 (1978), 329-336.

[4] H. Wielandt, Finite permutation groups (Academic Press, New York-London, 1964).

\section{Department of Mathematics}

Imperial College

London S.W.7

England 